\title{
Massage therapy for cardiac surgery patients-a randomized trial
}

\author{
Lesley A. Braun, PhD, ${ }^{\text {a,b }}$ Catherine Stanguts, BNurs, BHSc, ${ }^{a}$ Lisa Casanelia, BHSc, Grad Cert Ed, \\ Ondine Spitzer, MSocHlth, ${ }^{\mathrm{a}}$ Eldho Paul, MSc, ${ }^{\mathrm{d}}$ Nicholas J. Vardaxis, $\mathrm{PhD},{ }^{\mathrm{c}}$ and \\ Franklin Rosenfeldt, MBBS, FRACS ${ }^{\mathrm{a}}$
}

\begin{abstract}
Objectives: To determine whether massage significantly reduces anxiety, pain, and muscular tension and enhances relaxation compared with an equivalent period of rest time after cardiac surgery. The feasibility of delivering the treatment, effects on heart rate, blood pressure, and respiratory rate, and patient satisfaction were also assessed.
\end{abstract}

Methods: Elective cardiac surgery patients were randomized to receive massage or rest time at 2 points after surgery. Visual analog scales were used to measure pain, anxiety, relaxation, muscular tension, and satisfaction. Heart rate, respiratory rate, and blood pressure were measured before and after treatment. Focus groups and feedback were used to collect qualitative data about clinical significance and feasibility.

Results: A total of 152 patients (99\% response rate) participated. Massage therapy produced a significantly greater reduction in pain $(P=.001)$, anxiety $(P<.0001)$, and muscular tension $(P=.002)$ and increases in relaxation $(P<.0001)$ and satisfaction $(P=.016)$ compared to the rest time. No significant differences were seen for heart rate, respiratory rate, and blood pressure. Pain was significantly reduced after massage on day 3 or $4(P<.0001)$ and day 5 or $6(P=.003)$. The control group experienced no significant change at either time. Anxiety $(P<.0001)$ and muscular tension $(P<.0001)$ were also significantly reduced in the massage group at both points. Relaxation was significantly improved on day 3 or 4 for both groups (massage, $P<.0001$; rest time, $P=.006)$, but only massage was effective on day 5 or $6(P<.0001)$. Nurses and physiotherapists observed patient improvements and helped facilitate delivery of the treatment by the massage therapists on the ward.

Conclusions: Massage therapy significantly reduced the pain, anxiety, and muscular tension and improves relaxation and satisfaction after cardiac surgery. (J Thorac Cardiovasc Surg 2012;144:1453-9)

\section{Supplemental material is available online.}

Recovery from cardiac surgery is associated with severe chest pain at the site of sternotomy and limb pain at the site of conduit harvesting. ${ }^{1}$ Although pharmaceutical analgesia is standard postoperative care, pain and tenderness are still present, and drug side effects contribute further to the discomfort.

Mild state anxiety and depression also occur during the perioperative period and are associated with an increased risk of hospital readmission after coronary artery bypass

\footnotetext{
From the Cardiothoracic Surgical Research Unit, ${ }^{\text {a }}$ Department of Surgery, Monash University Alfred Hospital, Melbourne, Victoria, Australia; Pharmacy Department, ${ }^{\mathrm{b}}$ Alfred Hospital, Melbourne, Victoria, Australia; Academic Division, ${ }^{\mathrm{c}}$ Endeavour College of Natural Health, Melbourne, Victoria, Australia; and Department of Epidemiology and Preventive Medicine, ${ }^{\mathrm{d}}$ Monash University School of Public Health and Preventive Medicine, Melbourne, Victoria, Australia. Disclosures: Authors have nothing to disclose with regard to commercial support.

Received for publication Nov 8, 2011; revisions received March 22, 2012; accepted for publication April 3, 2012; available ahead of print Sept 10, 2012.

Address for reprints: Lesley A. Braun, PhD, Pharmacy Department, Alfred Hospital, Lower Ground Floor, Commercial Road, Prahran, Melbourne, Victoria 3181, Australia (E-mail: 1.braun@alfred.org.au).

$0022-5223 / \$ 36.00$

Crown Copyright $\odot 2012$ Published by Elsevier Inc. on behalf of The American Association for Thoracic Surgery

http://dx.doi.org/10.1016/j.jtcvs.2012.04.027
}

graft surgery. ${ }^{2}$ Massage therapy is a safe, noninvasive therapy that has been investigated in various populations and found to have significant effects in reducing pain, anxiety, blood pressure, and heart rate. ${ }^{3}$ As such, it might act as a useful nonpharmacologic adjunct to standard postoperative treatment of cardiac surgery patients.

Massage therapy encompasses an assortment of techniques involving manual manipulation of the soft tissues of the body through pressure and movement. It has the potential to reduce pain and discomfort and also psychological stress and anxiety. Five clinical studies have been conducted with cardiac surgery patients using massage therapy, three of which used massage as a standalone treatment, but the results have been conflicting, leaving many questions unanswered. ${ }^{4-8}$

The primary aims of the present study were to determine whether massage therapy produces a significant reduction in patient-reported pain, anxiety, and muscular tension (primary outcomes) compared with an equivalent period of rest time. The secondary aims were to determine whether massage therapy produces a significant change in the heart rate, blood pressure, and respiratory rate (secondary outcomes) compared with those after rest time, enhances patients' sense of relaxation and satisfaction with their hospital stay, and is feasible to be delivered in an acute care setting. 


\section{Abbreviation and Acronym}

VAS $=$ visual analog scale

\section{METHODS}

The present prospective randomized study was conducted at the Alfred Hospital (Melbourne, Victoria, Australia) between April 2009 and January 2011. Cardiothoracic surgical patients were considered eligible for inclusion in the present study if they were elective patients scheduled to undergo coronary artery bypass graft surgery or valve surgery, or both, older than 18 years, mentally alert, and could speak English. Patients were invited to participate in the study during their preadmission visit and, if they agreed, had the study explained to them before signing a participant consent form. After consent, the patients were randomly assigned to receive massage therapy or an equivalent period of rest time (active control) on the ward on day 3 or 4 and again on day 5 or 6 after surgery. The randomization scheme used computergenerated, blocked numbers to ensure a balanced allocation throughout the study period. A flow diagram of the study is shown in Figure 1.

\section{Measures}

Visual analog scales (VASs) were used to evaluate the patients' perception of pain, anxiety, muscular tension, relaxation, and satisfaction before and after each intervention. The VASs were labeled no pain (score, 0 ) to worst pain ever (score, 10), no anxiety (score, 0) to worst anxiety ever (score, 10), no muscular tension (score, 0 ) to extreme tension (score, 10), complete relaxation (score, 10) to none (score, 0 ), and overall satisfaction with their allocated intervention from not satisfied (score, 0 ) to very satisfied (score, 10). The VAS used to measure pain was accompanied by a diagram of faces depicting different pain levels. Patients placed a mark on the scale to reflect their rating at survey completion, which was then collected by a research nurse after it was finished. A cardiac nurse measured the patients' blood pressure and heart rate using an automatic monitoring device and manually measured the respiratory rate before and after the patients' designated intervention. The measures were taken immediately before each intervention (within 10 minutes) and immediately after (within 10 minutes).

The massage therapists recorded the primary areas of patient-reported discomfort before and after massage and spontaneous patient feedback, as well as data on whether patients had received a massage before, where the massage took place (chair or bed; private or shared room), and any issues or problems encountered during therapy. Data collection for both groups occurred at the same time each day, from 12 to 4 PM, to control for activity variables.

To explore the feasibility of providing massage therapy on the wards, the massage therapists kept personal notes describing their observations, and 2 focus groups were conducted with nursing and physiotherapy staff and massage therapists in July 2009 and August 2010. At these group sessions, verbal feedback was collected about the staff

$\mathrm{n}=152$ consented ( $99 \%$ response rate) and randomised

6 did not receive intervention due to cancelled surgery

or still on waiting list at time of study completion
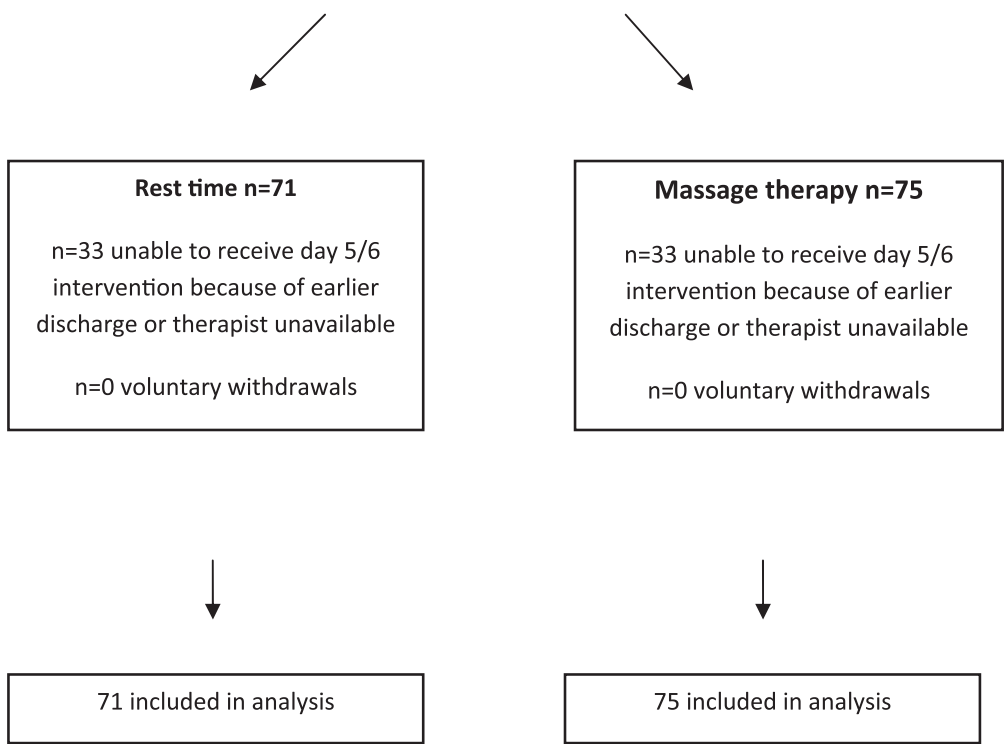

FIGURE 1. Flow diagram of patient recruitment and treatment. 
TABLE 1. Patient characteristics $(n=152)$

\begin{tabular}{lccr}
\hline \multicolumn{1}{c}{ Variable } & $\begin{array}{c}\text { Active } \\
(\mathbf{n = 7 6 )}\end{array}$ & $\begin{array}{c}\text { Control } \\
(\mathbf{n}=\mathbf{7 6})\end{array}$ & $\begin{array}{r}\boldsymbol{P} \\
\text { value }\end{array}$ \\
\hline Male gender & $51(67 \%)$ & $62(82 \%)$ & .04 \\
Age (y) & $66.8 \pm 11.3$ & $66.6 \pm 11.7$ & .93 \\
Surgical procedure & & & \\
$\quad$ CABG only & $37(49 \%)$ & $40(53 \%)$ & .63 \\
Valve only & $26(34 \%)$ & $22(29 \%)$ & .49 \\
CABG and valve & $10(13 \%)$ & $11(14 \%)$ & .81 \\
CABG, valve, and/or other & $3(4 \%)$ & $3(4 \%)$ & 1.00 \\
Hypertension & 61 & 56 & \\
Diabetes & 13 & 17 & \\
Readmitted within 30 d after surgery & 9 & 10 & \\
ICU stay (h) & & & \\
$\quad$ Median & 47.0 & 47.5 & \\
Average & 57.58 & 56.18 & \\
Length of stay (d) & & & \\
Median & 7.5 & 8.0 & \\
Average & 9.6 & 9.125 & \\
\hline
\end{tabular}

Data presented as $\mathrm{n}(\%)$ or mean \pm standard deviation. $C A B G$, Coronary artery bypass grafting; $I C U$, intensive care unit.

and therapists' observations of participating patients and a discussion was held of the practical issues relating to therapy implementation and staff communication.

\section{Sample Size}

From the available published data, a sample size of 50 patients per group was determined as being able to provide $80 \%$ power to detect a difference in the pain score equivalent to approximately $56 \%$ of 1 standard deviation in continuous, normally distributed outcomes between the massage therapy and rest time groups at $P<.05$. Translated into the absolute difference, this effect equated approximately to a 1-point difference on the VAS. We increased the sample size by $50 \%$ to account for unavoidable patient withdrawal, such as early discharge or extended stays in the intensive care unit.

\section{Intervention}

A massage therapy coordinator supervised the work of the therapists and provided a guide to the main techniques to be used. Therapists adapted these according to the patient's presentation and preferences. Eligible patients received 20 minutes of individualized therapy in their room and could choose to stay in bed semireclined or sit on the edge of their bed or in a chair, after visitors had been asked to leave the room. Therapists explained the nature of the intervention before beginning and positioned patients according to their individual needs and limitations. Patients were given a choice of areas to be massaged, such as shoulders, back, neck, scalp, hands, feet, or legs. The location of the massage was usually the area of maximal discomfort as determined by the patient. Therapists provided treatment mostly using Swedish massage techniques applied with moderate pressure and using gliding and kneading movements. Particular attention was given to avoiding sites at which medical apparatus was attached or surgical wounds were present.

Unscented sorbolene and glycerine cream was applied to the skin as a lubricant because this is a hypoallergenic preparation readily available on the wards and removed the possibility of scented aromatherapy oils confounding the treatment effects. Treatment was provided free of charge to the study participants.

\section{Active Control Group}

The patients allocated to receive 20 minutes of rest time were encouraged to spend their allocated time relaxing quietly in a chair or bed. The visitors were asked to leave during this period, and a sign was posted on the door or curtain asking the staff not to disturb the patient. Patient medications and the usual tests were not administered during this period to ensure that the patients were not disturbed.

The Alfred Hospital Human Research and Ethics Committee provided ethics approval for the present study. The present randomized controlled trial followed the principles outlined in the Consolidated Standards of Reporting Trials (CONSORT) statement and has been registered in the Australian New Zealand Clinical Trials Registry (registration number ACTRN12610000191099).

\section{Statistical Analysis}

Differences in proportions between groups were compared using the $\chi^{2}$ test for equal proportions or Fisher's exact test if the numbers were small. Normally distributed variables were compared using Student $t$ test and nonnormally distributed variables were compared using the Wilcoxon rank sum test. Changes in the VAS scores (anxiety, pain, relaxation, satisfaction, and tension) were assessed using repeated measures analysis of variance, fitting a group effect, a time effect, and an interaction between group and time, with results reported as the adjusted mean \pm standard error. Descriptive and inferential statistics were calculated using SAS, version 9.2 (SAS Institute, Cary, NC).

\section{RESULTS}

\section{Patients}

During the study period, 154 eligible surgical patients attending the preadmission clinic were invited to participate in the present study. Of these, 152 agreed to participate (99\% response rate). The patient characteristics are listed in Table 1. During the study period, 146 patients received either massage therapy or an equivalent period of rest time. Of those allocated to receive massage, 58\% reported it would be their first time receiving massage therapy.

\section{Pain}

Massage delivered on day 3 or 4 significantly reduced the patients' average pain score (from $2.9 \pm 0.3$ to $1.4 \pm$ $0.2 ; P<.0001$ ); a reduction of $52 \%$ (Figure 2 ). In contrast, the pain was not significantly reduced for patients receiving an equivalent period of rest time (from $2.3 \pm 0.2$ to $2.0 \pm$ $0.25 ; P=.21$ ). The change in pain scores for day 5 or 6 showed a similar pattern. Massage produced a $38 \%$ reduction in the average pain scores, from $2.1 \pm 0.35$ to $1.3 \pm 0.3$ $(P=.003)$, but no significant change was reported for the rest time group ( $1.7 \pm 0.3$ to $1.3 \pm 0.3 ; P=.20$; Table 2$)$.

For both measurement periods combined, massage therapy produced a significantly greater reduction in pain scores $(1.19 \pm 0.17)$ compared with rest time $(0.32 \pm 0.18$; $P=.001)$ and was the more effective treatment.

\section{Anxiety}

Massage delivered on day 3 or 4 reduced the anxiety scores by $58 \%$, from $3.6 \pm 0.4$ to $1.5 \pm 0.2(P<.0001)$. However, rest time delivered during the same period did not significantly change the anxiety scores (from $3.0 \pm$ 0.3 to $2.6 \pm 0.3 ; P=.22$; Figure 3 ). Massage delivered on day 5 or 6 again significantly reduced the anxiety scores, 


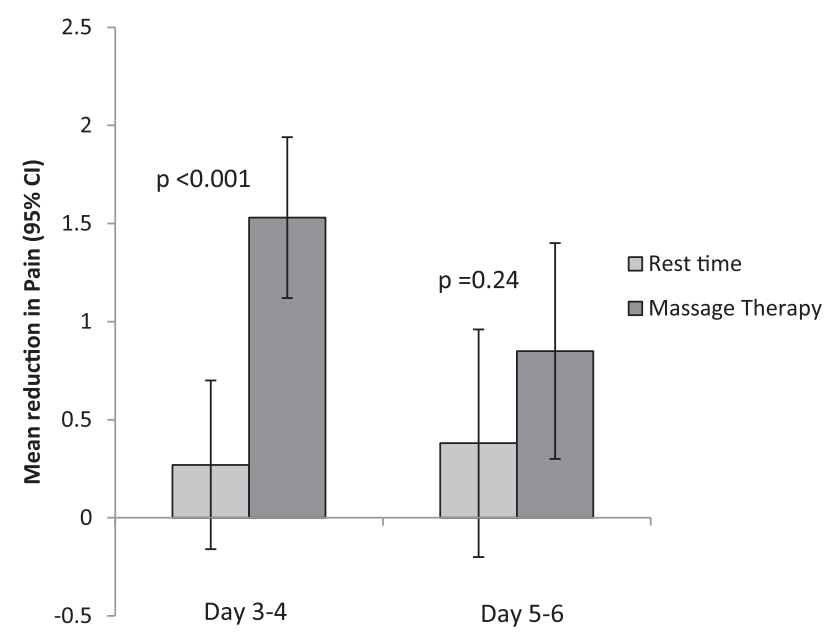

FIGURE 2. Adjusted mean change in visual analog scale scores for pain in massage therapy and rest time groups at 2 points. Error bars represent standard error of the mean. $C I$, Confidence interval.

from $3.5 \pm 0.4$ to $2.1 \pm 0.4(P<.0001)$, a $40 \%$ reduction compared with rest time therapy, which had no significant effect (from $3.6 \pm 0.4$ to $3.1 \pm 0.5 ; P=.19$ ).

For both periods combined, massage was shown to be superior to rest time for reducing anxiety scores, with massage therapy reducing the anxiety scores by $1.72 \pm 0.23 \mathrm{com}$ pared with rest time $(0.41 \pm 0.24 ; P<.0001$; Table 2$)$.

\section{Relaxation}

Massage therapy delivered on day 3 or 4 significantly increased the mean relaxation scores (from $6.0 \pm 0.3$ to $8.7 \pm 0.2 ; P<.0001)$ and again on day 5 or $6(7.1 \pm 0.4$ to $8.7 \pm 0.2 ; P<.0001)$, with increases of $45 \%$ and $23 \%$, respectively (Figure E1). Similarly, the rest time on day 3 or 4 resulted in a significant change in the mean relaxation scores (from $6.4 \pm 0.25$ to $7.2 \pm 0.3 ; P=.006$ ); however, on day 5 or 6 , the increase was not significant (from $6.4 \pm 0.4$ to $7.2 \pm 0.4 ; P=.074$ ).

For both treatment periods combined, massage was shown to be superior to rest time for improving the relaxation scores with massage improving the scores by

TABLE 2. Comparison between treatment groups of changes in variables from before to after intervention, averaged for 2 periods (days 3 or 4 and 5 or 6 )

\begin{tabular}{lccc}
\hline \multirow{2}{*}{ Variable } & \multicolumn{2}{c}{ Intervention } & \\
\cline { 2 - 3 } & Massage therapy & Rest time & P value \\
\hline Pain & $1.19 \pm 0.17$ & $0.32 \pm 0.18$ & .001 \\
Anxiety & $1.72 \pm 0.23$ & $0.41 \pm 0.24$ & $<.0001$ \\
Muscle tension & $1.70 \pm 0.24$ & $0.61 \pm 0.25$ & .002 \\
Relaxation & $2.11 \pm 0.23$ & $-0.74 \pm 0.25$ & $<.0001$ \\
Satisfaction & $0.31 \pm 0.17$ & $-0.28 \pm 0.17$ & .016 \\
\hline
\end{tabular}

Data presented as mean \pm standard error.

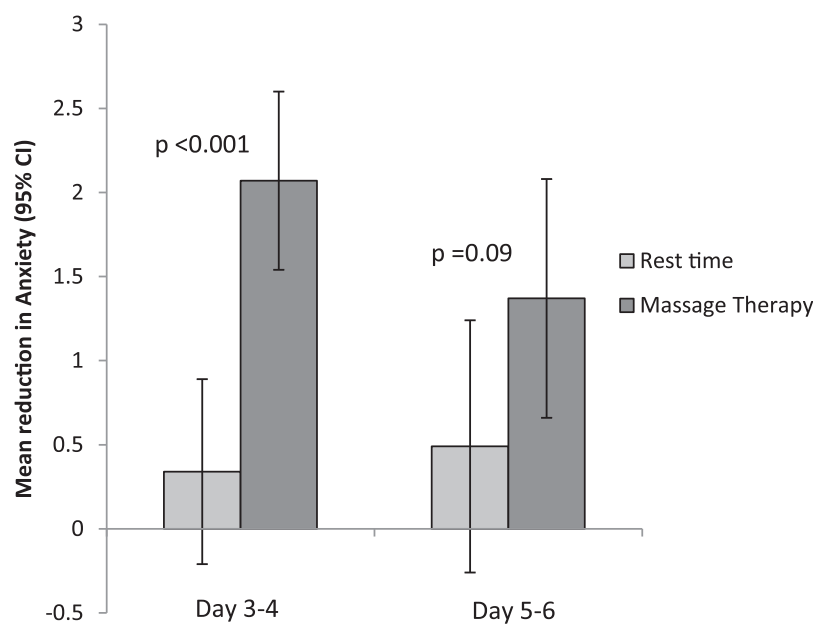

FIGURE 3. Adjusted mean change in visual analog scores for anxiety in massage therapy and rest time groups at 2 points. Error bars represent standard error of the mean. $C I$, Confidence interval.

$2.11 \pm 0.23$ compared with rest time $(0.74 \pm 0.25$; $P<.0001$; Table 2).

\section{Muscular Tension}

Massage administered on day 3 or 4 significantly reduced the mean muscular tension scores from $3.7 \pm 0.3$ to $1.7 \pm$ $0.2(P<.0001)$, a reduction of $54 \%$ (Figure E2). Similar results were obtained for massage delivered on day 5 or 6 , which reduced the muscular tension scores from $3.2 \pm$ 0.3 to $1.8 \pm 0.3(P<.0001)$, a $44 \%$ reduction.

The rest time did significantly reduce the muscular tension scores on day 3 or 4 , from $3.3 \pm 0.3$ to $2.6 \pm$ $0.3(P=.01)$ but did not have a significant effect on day 5 or 6 (score change from $3.3 \pm 0.4$ to $2.8 \pm 0.4$; $P=.19$ ).

For both periods combined, the data confirmed that massage resulted in a greater reduction in muscular tension scores $(1.70 \pm 0.24)$ than rest time $(0.61 \pm 0.25)$ and was the more effective treatment when the results were compared $(P=.002$; Table 2$)$.

\section{Satisfaction}

For patients' satisfaction with their hospital stay, a significant increase was reported on day 3 or 4 after massage therapy $(P=.03)$, although the rest time was associated with a nonsignificant trend toward dissatisfaction (Figure E3). No significant changes were seen in either group on day 5 or 6 .

For both periods combined, massage produced a greater improvement in satisfaction than rest time $(0.31 \pm 0.17$ vs $-0.28 \pm 0.17 ; P=.016$; Table 2 ). The effect of massage therapy on blood pressure, heart rate and respiratory rate, and systolic and diastolic blood pressure, were reduced in the intervention and control groups after each treatment, 
with no significant between-group differences for any of these 4 variables.

\section{Massage Therapy Delivered}

Overall, 119 massage treatments were administered. Of these, $47 \%$ were delivered to patients in their beds and $53 \%$ to patients in a chair, with no significant differences seen in the proportion of patients receiving treatment in their bed or chair between day 3 or 4 and day 5 or 6 . Information about the physical areas where patients received massage treatment (multiple responses received) is listed in Table E1.

\section{Feasibility and Acceptance Among Staff}

Two focus group discussions were held during the study course. The participants included the massage therapists and key nursing staff. Both nurses and therapists commented on how well the interventions had been accepted by the patients and staff and how implementing treatment on the ward had been relatively easy.

Nurses commented that it was easy to incorporate a time for massage therapy into the daily routines of patient care. They reported that the patients were excited about the treatments offered and were often more relaxed and less anxious as a result of receiving the massage. Massage therapists reported excellent patient feedback generally, noting that any initial patient apprehension was overcome by the second intervention.

The chief ward physiotherapist was also interviewed twice during the study period and was very enthusiastic about the benefits of the massage therapy, commenting that the physiotherapists' role has become more medically focused and that they have little time to deliver such treatments on a busy ward.

As a result of considerable nursing and physiotherapy interest during the study, the massage therapists received 91 additional referrals for patients outside the study, predominantly from the physiotherapy department.

\section{DISCUSSION}

The results from the present randomized study have shown that massage therapy can be safely and effectively delivered on a busy cardiothoracic surgical ward, with no increase in adverse events, significant reductions in pain, anxiety, and muscular tension, and enhancement of patients' sense of relaxation. Furthermore, we have shown that although many patients had never received massage before, they still experienced significant benefits suggesting that previous experience, or preparatory knowledge about the expected procedure, is unnecessary.

Massage therapists working on the wards and the treatment they delivered were well accepted by the nursing and physiotherapy staff. The high patient participation rate, lack of voluntary withdrawals from the study, and improved patient satisfaction immediately after the treatment have also confirmed patient acceptance of massage in the hospital setting.

\section{Massage Research and Cardiac Surgery Patients}

Five clinical studies have been conducted of cardiac surgery patients using massage therapy but produced conflicting results. ${ }^{4-8}$ A pilot study of 56 patients by Cutshall and colleagues ${ }^{6}$ at the Mayo Clinic in Rochester found 20 minutes of massage therapy produced a significant reduction in pain and anxiety in cardiac surgery patients compared with 20 minutes of quiet time. These findings were subsequently confirmed in a larger randomized study conducted by the same group. ${ }^{5}$ In contrast, a randomized study by Albert and colleagues ${ }^{4}$ from the Cleveland Clinic found no significant differences between massage therapy and usual care for any tested variables, including pain, anxiety, and depression. Two other studies used massage therapy, together with other treatments, such as aromatherapy, guided imagery, or music. ${ }^{7,8}$

In our study, the first treatment was provided on day 3 or 4, the period when most patients have had their intravenous lines and tubes removed, and massage therapists can more easily provide treatment without these obstructions. Previously, Bauer and colleagues ${ }^{5}$ reported that postoperative massage given on day 2 increased patients' pain on day 3 . They suggested that patients' grogginess and the presence of the intravenous lines and tubes might have compromised their ability to experience the full benefits of treatment. ${ }^{5}$ Massage therapy provided in the study by Albert and colleagues ${ }^{4}$ was also delivered earlier, on day 2 or 3 , and found to be ineffective in reducing pain.

Another contributor to our positive results relates to the actual massage treatment itself. Unlike the previous negative study, ${ }^{4}$ in which patients were turned onto their left side when massage was performed, our patients were sitting up in bed or forward in a chair; thus, no extra pressure was experienced near the sternal wound. Additionally, massage therapy was individualized to meet each patient's needs. Instead of following a strict regimen of predefined steps, the massage therapists were given a guide to treatment that was discussed with the patient. Throughout the session, patient feedback guided the therapists' use of pressure and choice of technique. This method of patient-therapist discussion and feedback more closely aligns with how treatment is delivered outside of the patient/hospital setting and is in accordance with the principles of patientcentered care.

The effect of interpersonal attention as a contributing factor to the benefits of massage should also be considered when interpreting these results. It seems reasonable to expect that patients in a busy ward will gain benefit from the attention of a therapist whose main aim is to provide comfort. Although we did not measure the effects of 
personal attention on the treatment outcomes and therefore could not state its contributory effect, a previous study comparing the effect of personal attention by a nurse to the effect of massage after cardiac surgery confirmed that massage was superior for relaxation and well being, thereby discounting the attention effect as a major contributor to the results achieved. ${ }^{7}$

\section{How Does Massage Achieve Therapeutic Effects?}

The physical discomfort experienced by cardiac surgery patients results from a variety of factors, including trauma and manipulation of the body during surgery, the immobility for several days afterward, and the physical manifestations of psychological stress. Massage therapy addresses both the physical and psychological symptoms by reducing muscular tension and pain and decreasing anxiety and increasing relaxation. Most patients received localized treatment to their neck and shoulders, which was sufficient to reduce their overall perception of anxiety and relaxation, suggesting a systemic response to a locally applied treatment.

The mechanisms behind the clinical effectiveness of massage therapy are not well established, and no single mechanism can account for the myriad effects reported after massage. It is likely that massage therapy works as a result of multiple mechanisms. A model proposed by Bialosky and colleagues ${ }^{9}$ suggests that a mechanical force from massage therapy initiates a cascade of neurophysiologic responses from the peripheral and central nervous system that are then responsible for the clinical outcomes.

Traditionally, the pain-relieving effects of massage have been explained using the gate-control theory of pain transmission and modulation. ${ }^{10}$ This theory suggests that the experience of pain can be modified by the application of competing stimuli. Thus, the physical pressure applied with massage travels down the nerve pathways faster than those transmitting the sensation of pain, thereby blocking pain transmission. ${ }^{11}$

Another theory proposes that massage therapy activates the autonomic parasympathetic response, thereby reducing the "fight or flight" stress response and inducing a state of decreased cardiovascular activity and increased calmness and relaxation. ${ }^{3}$ In the absence of measuring biomarkers such as adrenaline and noradrenaline, we measured changes in blood pressure, heart rate, and respiratory rate as surrogate markers of parasympathetic activation and/or sympathetic deactivation. From our results, it appears that massage therapy is likely to activate the autonomic nervous system in cardiac surgery patients, but only to the same extent as an equivalent period of rest time.

A small functional neuroimaging study involving healthy volunteers showed that massage therapy delivered by a trained practitioner activated the cerebral reward centers, which correspond with known dopamine projections sites, triggering the association of a pleasurable sensation with massage therapy. ${ }^{12}$ Additional research with larger patient numbers is required to confirm these results.

\section{Study Strengths and Limitations}

Many unique challenges exist to conducting rigorous research on massage therapy, including blinding patients to treatment, finding an acceptable control intervention, and avoiding self-selection bias by participants who agree to participate in such research. To overcome some of these limitations, we chose to use as our comparator intervention an equivalent amount of rest time, because it was thought that patients could feasibly believe this to have pain- and stressrelieving benefits. The study was described to patients at all times as a stress reduction study, instead of a massage study to avoid self-selection bias; however, blinding was impossible, and the patients were aware of the intervention received.

In the present study, we did not evaluate for a reduction in analgesic medication usage. This was because the postoperative ward had a strict protocol for analgesic medication administration given to prevent pain and encourage mobilization. We believe that the massage programs could allow earlier weaning from the protocol and thus reduce the side effects of the medication and promote earlier mobilization.

The main strengths of the present study were the use of randomization to allocate patients to an active control group or massage therapy, the use of both quantitative and qualitative methods of data collection, and the excellent patient response rate.

\section{CONCLUSIONS}

The combined results we have presented have confirmed that postoperative massage therapy delivered from day 3 onward to cardiac surgery patients represents a useful adjunct to standard care for relieving patient discomfort and providing both physical and psychological benefits to enhance their hospital stay. The widespread acceptance of the massage therapists and treatment intervention by patients and nursing and physiotherapy staff demonstrates that a permanent massage therapy service would be welcome on the wards and integration within the current standard patient care protocols achievable.

Particular thanks to Dr Ruchong Ou for assistance with the preparation of the diagrams.

\section{References}

1. Redeker NS. Symptoms reported by older and middle-aged adults after coronary bypass surgery. Clin Nurs Res. 1993;2:148-59.

2. Tully PJ, Baker RA, Turnbull D, Winefield $\mathrm{H}$. The role of depression and anxiety symptoms in hospital readmissions after cardiac surgery. J Behav Med. 2008;31: 281-90.

3. Moyer CA, Rounds J, Hannum JW. A meta-analysis of massage therapy research. Psychol Bull. 2004;130:3-18.

4. Albert NM, Gillinov AM, Lytle BW, Feng J, Cwynar R, Blackstone EH. A randomized trial of massage therapy after heart surgery. Heart Lung. 2009;38: 480-90. 
5. Bauer BA, Cutshall SM, Wentworth LJ, Engen D, Messner PK, Wood CM, et al. Effect of massage therapy on pain, anxiety, and tension after cardiac surgery: a randomized study. Complement Ther Clin Pract. 2010;16:70-5.

6. Cutshall SM, Wentworth LJ, Engen D, Sundt TM, Kelly RF, Bauer BA. Effect of massage therapy on pain, anxiety, and tension in cardiac surgical patients: a pilot study. Complement Ther Clin Pract. 2010;16:92-5.

7. Stevenson C. Measuring the effects of aromatherapy. Nurs Times. 1992;88: 62-3.

8. Kshettry VR, Carole LF, Henly SJ, Sendelbach S, Kummer B. Complementary alternative medical therapies for heart surgery patients: feasibility, safety, and impact. Ann Thorac Surg. 2006;81:201-5.
9. Bialosky JE, Bishop MD, Price DD, Robinson ME, George SZ. The mechanisms of manual therapy in the treatment of musculoskeletal pain: a comprehensive model. Manual Ther. 2009;14:531-8.

10. Melzack R, Wall PD. Pain mechanisms: a new theory: a gate control system modulates sensory input from the skin before it evokes pain perception and response. Pain Forum. 1996;5:3-11.

11. Wang AT, Sundt TM III, Cutshall SM, Bauer BA. Massage therapy after cardiac surgery. Semin Thorac Cardiovasc Surg. 2010;22:225-9.

12. Ho YWL, Higuchi S, Roberts N, Nurmikko T. Brain reward activity during massage: a functional neuroimaging investigation. NeuroImage. 2009;47(Suppl 1): S160 


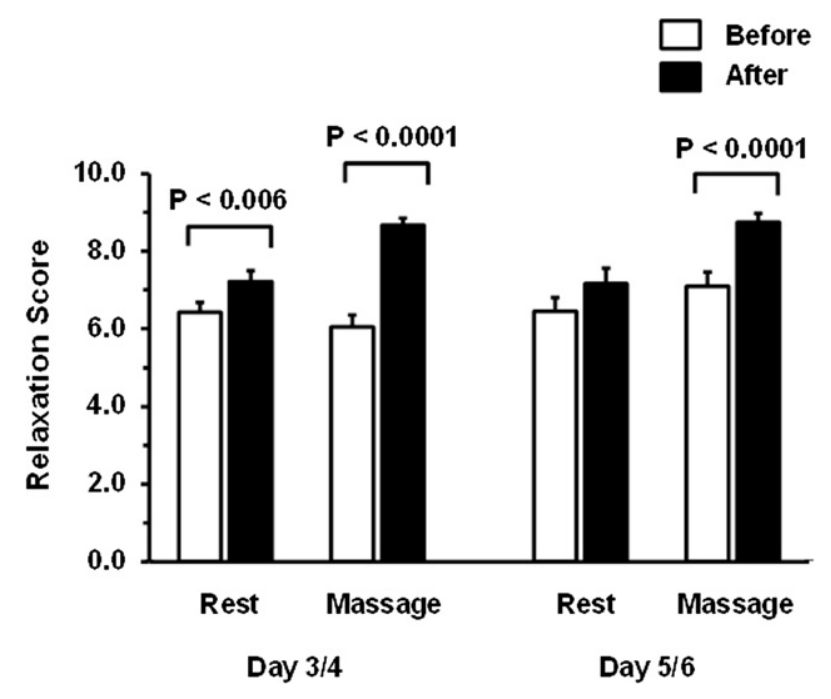

FIGURE E1. Relaxation scores for massage therapy and rest time at 2 points. Error bars represent standard error of the mean.

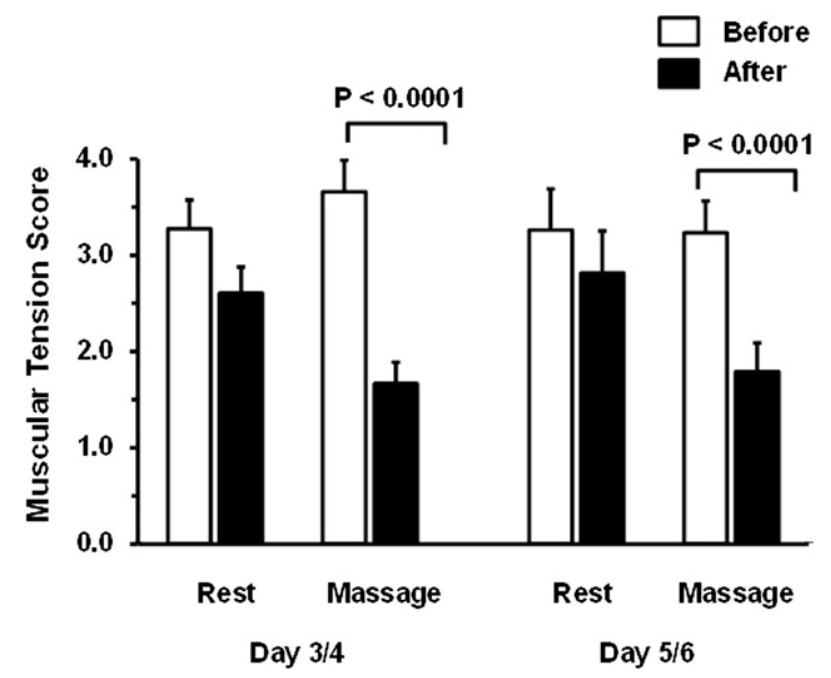

FIGURE E2. Muscular tension scores for massage therapy and rest time at 2 points. Error bars represent standard error of the mean.

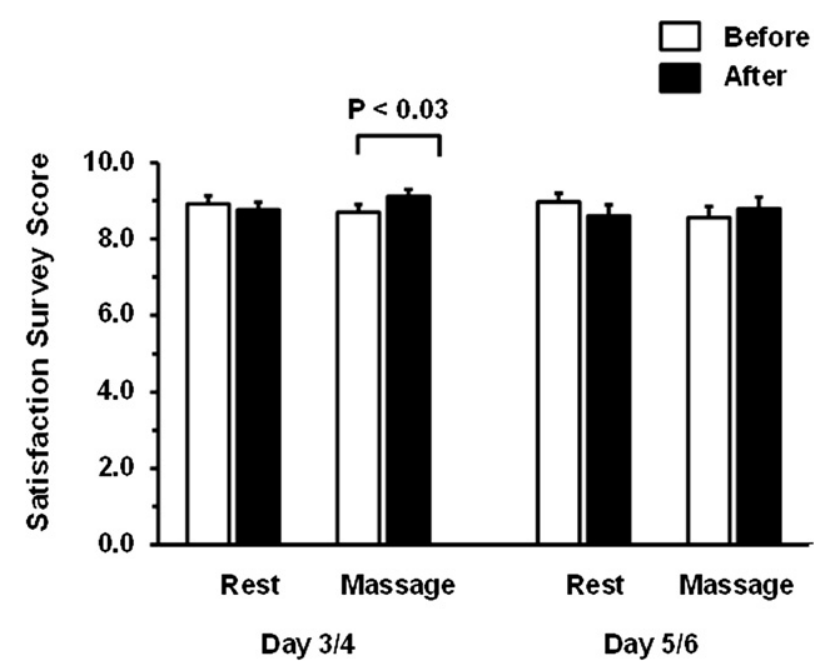

FIGURE E3. Satisfaction survey scores for massage therapy and rest time at 2 points. Error bars represent standard error of mean.
TABLE E1. Body locations where massage was applied to patients

\begin{tabular}{ll}
\hline Location & $\mathbf{n}(\%)$ \\
\hline Shoulders & $80(67)$ \\
Neck & $72(61)$ \\
Back & $60(50)$ \\
Head & $33(28)$ \\
Arms & $24(20)$ \\
Feet & $13(11)$ \\
\hline Percentage is of total treatments delivered.
\end{tabular}

